\title{
Stressed web environments as strategic games: Risk profiles and Weltanschauung *
}

\author{
J. Gabarro ${ }^{(1)}$, P. Kilpatrick ${ }^{(2)}$, M. Serna ${ }^{(1)}$, and A. Stewart ${ }^{(2)}$ \\ (1) ALBCOM. LSI Dept. Universitat Politècnica de Catalunya, Barcelona. \\ (2) School of Computer Science. The Queen's University of Belfast, Belfast. \\ \{gabarro,mjserna\}@lsi.upc.edu \\ $\{$ a.stewart,p.kilpatrick\}@qub.ac.uk
}

\begin{abstract}
We consider the behaviour of a set of services in a stressed web environment where performance patterns may be difficult to predict. In stressed environments the performances of some providers may degrade while the performances of others, with elastic resources, may improve. The allocation of web-based providers to users (brokering) is modelled by a strategic non-cooperative angel-daemon game with risk profiles. A risk profile specifies a bound on the number of unreliable service providers within an environment without identifying the names of these providers. Risk profiles offer a means of analysing the behaviour of broker agents which allocate service providers to users. A Nash equilibrium is a fixed point of such a game in which no user can locally improve their choice of provider - thus, a Nash equilibrium is a viable solution to the provider/user allocation problem. Angel daemon games provide a means of reasoning about stressed environments and offer the possibility of designing brokers using risk profiles and Nash equilibria.
\end{abstract}

\section{Introduction}

A web-based computation involves the discovery and utilisation of services. It is often the case that a service is made available by a number of providers. The performance of a provider can vary greatly over time (although service level agreements (SLAs) may provide information about "normal" expected performance). Brokers [2] are often used to monitor provider performance and to provide an interface to the "best" current provider.

It is usually the case that the performance of a provider deteriorates as demand increases (although "elastic" providers may call on extra servers in times of peak demand - thus, in such (stressed) situations performance can conceivably improve). The goal of this paper is to study the behaviour of a set of service providers in a stressed environment with the hope that a clearer understanding of stressed behaviour may aid the design of intelligent brokers.

\footnotetext{
* J. Gabarró and M. Serna are partially supported by FET pro-active Integrated Project 15964 (AEOLUS), TIN-2007-66523 (FORMALISM), TIN-2005-25859-E and SGR 2009-2015 (ALBCOM).
} 
Here we adopt the point of view that providers should be treated in toto since web users alternate between providers in times of high usage. We also assume that users behave in a non-cooperative way (in that the behaviour of others is, usually, irrelevant). Given these assumptions it is reasonable to model the behaviour of a set of providers (resources) in a stressed environment as a strategic situation in algorithmic game theory $[8,11,12]$. The notion of a Nash equilibrium is used to derive an efficient broker allocation of providers to users (see Example 1).

Example 1. Brokering in an idealised environment. Consider a situation where a set of users $\{1, \ldots, n\}$ submit jobs for execution to a broker. Suppose that the broker uses multiple predictable service providers (resources) $R=\left\{r_{1}, \ldots r_{k}\right\}$ to meet demand. The broker allocates service providers to jobs in such a way as to minimise user delay. This situation can be modelled by a non-cooperative game with $n$ players in which users "move" in sequence by allocating (or reallocating) their job to a provider. Providers may have modified work loads and delays as a consequence of a sequence of "moves". A Nash equilibrium is an allocation schedule in which no user can improve their situation by making a move ${ }^{1}$.

The web is comprised of a very diverse range of resources. Such heterogeneity contributes to the complexity of a web environment. Performance variability and sporadic unavailability of underlying networks provide further complications. Conventionally, unreliability is treated from a probabilistic viewpoint $[9,1,10]$. In contrast, we investigate a variety of provider behaviours within a stressed web environment using non-cooperative game theory (see Example 2).

Example 2. Brokering in a stressed environment. Now consider a more realistic refinement of the brokering example 1 where provider and network behaviour is less predictable. The following assumptions are made about stressed web environments:

1. stress is non-uniformly distributed across the web;

2. patches of stress can move dynamically in response to users moving jobs from stressed regions to more responsive providers;

3. the performance of certain providers may be highly vulnerable to heavy work loads; other providers may incorporate autonomic behaviour which increases the number of servers on offer in response to increased demand (elasticity). Consequently, some providers may be associated with increased unreliability at times of stress while others may exhibit robust behaviour.

An extended form of non-cooperative game is used to reason about brokering in stressed environments; in addition to the $n$ users the game additionally contains two extra players: a daemon player who selects a number of sites to be stressed so as to maximise the delay associated with the game (in a sense the daemon player

\footnotetext{
${ }^{1}$ A set of users may individually find service providers without utilising a broker; this process also corresponds to a game which may reach a Nash equilibrium. The broker model acts as an abstraction of this alternative allocation problem.
} 
models the deterioration of a stressed network at a set of vulnerable points); and an angel who selects a number of sites so as to minimise delay (in some sense the angel models the capacity of a network to modify its behaviour so as to improve throughput). Unreliability is described by the notion of a risk profile which specifies (a priori) possible angel and daemon behaviours; given a risk profile the behaviour of a broker in a stressed environment can be described by an associated $n+2$ player game.

Risk profiles were introduced in [3-5] to analyse network behaviour when a bounded number of services failed. However, risk profiles are sufficiently rich to allow the analysis of stressed web environments where networks may be under the influence of competing tendencies (one destructive, the other self-correcting). In a risk profile bounds are placed on both the constructive and destructive capacities (for example, an unreliable network may have no self-correcting behaviour and no angel player). As far we know the use of risk profiles to model stressed web environments is new.

The paper is structured as follows. Basic models of resource allocation games are given in $\S 2$, with particular attention placed on uniform unit allocation games. In $\S 3$ risk profiles are defined and Weltanschauungs are used to model stress in allocation problems. In $\S 4$ angel-daemon games are defined and used to analyse stressed resource allocation problems. Snapshots are used in $\S 5$ to provide a condensed description of strategy profiles; these are used to study pure Nash equilibria. In $\S 6$ we consider the structure of pure Nash equilibria in angeldaemon games. In $\S 7$ the idea of a risk-aware broker is developed. Finally in $\S 8$ some open questions are raised.

\section{Resource allocation games}

We consider a basic resource allocation game introduced by Koutsoupias and Papadimitriou in $[11,8]$ as a means of modelling simple competitive situations.

A resource allocation game (also called load balancing game) is a tuple

$$
C=\left\langle N, R,\left(w_{i}\right)_{i \in N},\left(d_{r}\right)_{r \in R},\left(A_{i}\right)_{i \in N},\right\rangle
$$

where the set of players is $N=\{1, \ldots, n\}$. Player $i \in N$ has to execute a job (or work) $w_{i}$. The set of resources is $R=\{1, \ldots, k\}$. Each resource $r \in R$ has a delay function $d_{r}$. For each player $1 \leq i \leq n, A_{i}$ is the set of possible allocations for work $w_{i}$, with $A_{i} \subseteq R$. A strategy $s_{i}$ (or action) for a player $i$ is an element of $A_{i}$, (player $i$ chooses a resource). A strategy profile is a tuple $s=\left(s_{1}, \ldots, s_{n}\right)$. Given a player $i$ and a strategy profile $s=\left(s_{1}, \ldots, s_{n}\right)$ we denote by $s_{-i}=\left(s_{1}, \ldots, s_{i-1}, s_{i+1}, \ldots, s_{n}\right)$ the profile where the strategy $s_{i}$ is missing. Given a strategy profile $s=\left(s_{1}, \ldots, s_{n}\right) \in A_{1} \times \cdots \times A_{n}$, the set of players using resource $r \in R$ is $L_{r}(s)=\left\{i \in N \mid r=s_{i}\right\}$ and the load of resource $r$ is the weight of the players using $r$, that is $\ell_{r}(s)=\sum_{i \in L_{r}(s)} w_{i}$. The cost for player $i$ of strategy profile $s$, is defined as $c_{i}(s)=d_{s_{i}}\left(\ell_{s_{i}}(s)\right)$. Following J. Bentham, 1748-1832, the social cost of a strategy profile $s$ is defined additively 
as $c_{\mathfrak{s}}(s)=\sum_{i \in N} c_{i}(s)$. In some examples, in order to simplify matters, costs are defined as $c_{i}(s)=\left\lceil d_{s_{i}}\left(\ell_{s_{i}}(s)\right)\right\rceil$ and $c_{\mathfrak{s}}(s)=\sum_{i \in N}\left\lceil d_{s_{i}}\left(\ell_{s_{i}}(s)\right)\right\rceil$.

Given a strategy profile $s=\left(s_{1}, \ldots, s_{n}\right)$ we define the load map of strategy $s$ as the vector $\ell(s)=\left(\ell_{1}(s), \ldots, \ell_{k}(s)\right)$ which describes macroscopically the load of each resource under strategy profile $s$. Observe that in load maps part of the information contained in a strategy profile is lost: we do not know which player is using a given resource.

Resource allocation games are a particular case of strategic games [6]. Strategic games can be used to model non-cooperative behaviour: a solution to a game corresponds to identifying Nash equilibria [6]. A pure Nash equilibrium (PNE for short) is a strategy profile $s^{*}$ such that for any player $i \in N$ and for any strategy $s_{i} \in A_{i}, c_{i}\left(s^{*}\right) \leq c_{i}\left(s_{-i}^{*}, s_{i}\right)$, where $\left(s_{-i}^{*}, s_{i}\right)$ denotes the strategy profile in which $s_{i}^{*}$ is replaced by $s_{i}$.

We are interested in analysing some specific natural types of delay functions, non-negative and monotone, and in particular affine functions, that is $d_{r}(x)=$ $d_{r} x$ with $0<d_{r}<\infty$.

Example 3. Consider an allocation game Fortran\&MPI_Servers defined in Figure 1 . The web environment comprises four servers $R=\{1,2,3,4\}$ with delays $d_{1}=1 / 2, d_{2}=1 / 4, d_{3}=1 / 4$ and $d_{4}=1 / 8$. Cost functions and social cost are defined using ceiling functions. Resources 1,2,3 can execute Fortran programs. Resources 1,3,4 can execute MPI programs. There are 5 jobs to be executed (superindices are used to denote the type of a job).

$$
w_{1}^{\mathrm{MPI}}=10, w_{2}^{\mathrm{F}}=5, w_{3}^{\mathrm{F}}=6, w_{4}^{\mathrm{MPI}}=15, w_{5}^{\mathrm{F}}=3
$$

Thus job 1 can be executed using resources (servers) 1,2 and 3 etc. and so $A_{1}=A_{4}=\{1,3,4\}$ and $A_{2}=A_{3}=A_{5}=\{1,2,3\}$. Consider a strategy profile $s=(1,1,1,4,3)$, where $s_{5}=3$ denotes that "job $w_{5}^{\mathrm{F}}$ is mapped to resource 3 ". The load map of this profile is $\ell(s)=(21,0,3,15)$. In $s$ the cost of player 1 is high because $c_{1}(s)=\left\lceil\frac{1}{2}\left(w_{1}^{\mathrm{MPI}}+w_{2}^{\mathrm{F}}+w_{3}^{\mathrm{F}}\right)\right\rceil=11$, therefore player 1 has a strong incentive to move his job to another server with lower current cost. Suppose that player 1 moves to server 3 (denoted by $1 \stackrel{w_{M}^{\mathrm{MPI}} \rightarrow}{\rightarrow} 3$ ): the cost to player 1 improves from 11 to 4 and therefore $s$ is not a PNE. By performing a sequence of similar kinds of move we finally get a PNE $(3,2,3,4,1)$ with social cost 13 . This PNE is non-unique: for instance $(4,2,3,4,2)$ is a PNE having a social cost 14 .

In some cases (uniform) all the resources have the same capability. This forces $A_{i}=R$ for $1 \leq i \leq n$, and moreover all the resources have the same delay function. We are specially interested in uniform affine delays $d_{r}(x)=d x$, $0<d<\infty$. We distinguish the case where all players have the same unit weight works (or jobs), i.e. $w_{i}=1$ for all $i \in N$. Inspired by [11], let $U n i t_{n, k, d}$ be the unit resource allocation game with $n$ players, $k$ resources and uniform affine delays with coefficient $d$. For unit weight games load maps provide enough information to describe a family of "equivalent" strategy profiles. The following lemma captures a widely-observed result. 
Lemma 1. In the Unit $t_{n, k, d}$ game, a strategy profile $s=\left(s_{1}, \ldots, s_{n}\right)$ is a PNE, iff $\ell(s)$ has $n \% k$ resources with load $\lceil n / k\rceil$ and the remaining resources have load $\lfloor n / k\rfloor$ and the social cost is $(\lceil n / k\rceil n+\lfloor n / k\rfloor n \% k) d$.

\begin{tabular}{|c|c|c|c|c|}
\hline \multirow{6}{*}{ Fortran\&MPI_Servers } & \multicolumn{4}{|c|}{ Resources } \\
\hline & 1 & 2 & 3 & 4 \\
\hline & \multicolumn{4}{|c|}{ Services } \\
\hline & $\mathrm{F}, \mathrm{MPI}$ & \begin{tabular}{l|l}
$\mathrm{F}$ \\
\end{tabular} & F, MPI & MPI \\
\hline & \multicolumn{4}{|c|}{ Delay } \\
\hline & $1 / 2$ & $1 / 4$ & $1 / 4$ & $1 / 8$ \\
\hline \multicolumn{5}{|c|}{ Initial strategy } \\
\hline$s$ & $\begin{array}{c}w_{1}^{\mathrm{MPI}}=10 \\
w_{2}^{\mathrm{F}}=5 \\
w_{3}^{\mathrm{F}}=6\end{array}$ & & $w_{5}^{F}=3$ & $w_{4}^{\mathrm{MPI}}=15$ \\
\hline Cost & 11 & & 1 & 2 \\
\hline \multicolumn{5}{|c|}{ Moves } \\
\hline $1 \stackrel{w_{1}^{\mathrm{MPI}}}{\rightarrow} 3$ & $\begin{array}{l}w_{2}^{\mathrm{F}}=5 \\
w_{3}^{\mathrm{F}}=6\end{array}$ & & $\begin{array}{c}w_{1}^{\mathrm{MPI}}=10 \\
w_{5}^{\mathrm{F}}=3\end{array}$ & $w_{4}^{\mathrm{MPI}}=15$ \\
\hline Cost & 6 & & 4 & 2 \\
\hline $1 \stackrel{w_{3}^{\mathrm{F}}}{\rightarrow} 3$ & $w_{2}^{\mathrm{F}}=5$ & & $\begin{array}{c}w_{1}^{\mathrm{MPI}}=10 \\
w_{3}^{\mathrm{F}}=6 \\
w_{5}^{\mathrm{F}}=3\end{array}$ & $w_{4}^{\mathrm{MPI}}=15$ \\
\hline Cost & 3 & & 5 & 2 \\
\hline $3 \stackrel{w_{5}^{\mathrm{F}}}{\rightarrow} 1$ & $\begin{array}{l}w_{2}^{\mathrm{F}}=5 \\
w_{5}^{\mathrm{F}}=3\end{array}$ & & $\begin{array}{c}w_{1}^{\mathrm{MPI}}=10 \\
w_{3}^{\mathrm{F}}=6\end{array}$ & $w_{4}^{\mathrm{MPI}}=15$ \\
\hline Cost & 4 & & 4 & 2 \\
\hline $1 \stackrel{w_{2}^{\mathrm{F}}}{\rightarrow} 2$ & $\overline{w_{5}^{\mathrm{F}}=3}$ & $\overline{w_{2}^{F}}=5$ & $\begin{array}{c}w_{1}^{\mathrm{MPI}}=10 \\
w_{3}^{\mathrm{F}}=6\end{array}$ & $w_{4}^{\mathrm{MPI}}=15$ \\
\hline Cost & 2 & 2 & 4 & 2 \\
\hline $3 \stackrel{w_{3}^{\mathrm{F}}}{\rightarrow} 2$ & $w_{5}^{F}=3$ & $\begin{array}{l}w_{2}^{\mathrm{F}}=5 \\
w_{3}^{\mathrm{F}}=6\end{array}$ & $w_{1}^{\mathrm{MPI}}=10$ & $w_{4}^{\mathrm{MPI}}=15$ \\
\hline Cost & 2 & 3 & 3 & 2 \\
\hline
\end{tabular}

Fig. 1. Allocation game Fortran\&MPI_Servers. Servers 1 and 3 offer Fortran and MPI services. Server 2 offers Fortran services and server 4 offers MPI services. An initial allocation $s=(1,1,1,4,3)$ is displayed. A sequence of moves which end with the PNE $(3,2,2,4,1)$ is also displayed.

Proof. Consider a game Unit ${ }_{n, k, d}$ with $n$ unit jobs (or works) $w_{i}=1,1 \leq i \leq n$ and $r$ servers (or resources). The broker allocates $\lfloor n / k\rfloor$ jobs into each server. After that there remain $n \% k$ jobs to allocate. As $n \% k<n$, the broker spreads these into the different servers (one per server). After such allocation, there are $n \% k$ servers with load $\lceil n / k\rceil$ and $r-n \% k$ server with load $\lfloor n / k\rfloor$. We can easily 
prove that such allocation is a PNE. Moreover any other "type" of allocation is not a PNE. In such cases there exists an oveloaded server and any job in such a server has interest to migrate.

Let us consider the social cost. The $n \% k$ servers contribute a social cost of $(n \% d)\lceil n / k\rceil^{2} d$. The remaining $k-n \% k$ servers contribute a cost $(n-n \% k)\lfloor n / k\rfloor^{2} d$. The social cost is $d k\lfloor n / k\rfloor^{2}+d(n \% k)\left(\lceil n / k\rceil^{2}-\lfloor n / k\rfloor^{2}\right)$ A little thought allows us to see $(n \% k)\left(\lceil n / k\rceil^{2}-\lfloor n / k\rfloor^{2}\right)=(n \% k)(2\lfloor n / k\rfloor+1)$ and the social cost is rewritten as $d k\lfloor n / k\rfloor^{2}+d(n \% k)(2\lfloor n / k\rfloor+1)$. As $k\lfloor n / k\rfloor+n \% k=n$ we rewrite as $d\lfloor n / k\rfloor(n \% k)+d n \% k$. As $n \% k(\lfloor n / k\rfloor+1)=n \% k\lceil n / k\rceil$ we get the final expression.

\section{Risk profiles and Weltanschauung}

Risk profiles [5] are used to describe stressed environments in which two competing forces act on resources. Assume that the set of resources $R$ in an allocation game is partitioned into two subsets $\mathcal{A}$ and $\mathcal{D}$ such that $R=\mathcal{A} \cup \mathcal{D}$ and $\mathcal{A} \cap \mathcal{D}=\emptyset$.

Subset $\mathcal{A}$ is controlled by an agent $\mathfrak{a}$ called the angel; $\mathcal{A}$ is used to model resources which behave robustly under stress. When a resource $r$ is selected by the angel it runs under the angelic delay function $d_{r}^{\mathcal{A}}$. The angel can force angelic behaviour only for a limited number of resources $f_{\mathcal{A}}$. The angel's objective is to improve system behaviour as much as possible.

Subset $\mathcal{D}$ is controlled by another agent $\mathfrak{d}$ called the daemon that exhibits malicious behaviour. When $r$ is selected by the daemon it runs under the daemonic delay function $d_{r}^{\mathcal{D}}$. Again the daemon can affect only a limited number of resources, $f_{\mathcal{D}}$. The daemon's objective is to maximise system delay.

We summarize all these ideas into the following definition of risk profile:

Definition 1. Given $C=\left\langle N, R,\left(w_{i}\right)_{i \in N},\left(d_{r}\right)_{r \in R},\left(A_{i}\right)_{i \in N}\right\rangle$, a risk profile for $C$ is a tuple $\mathcal{R}=\left\langle C, \mathcal{A}, \mathcal{D}, f_{\mathcal{A}}, f_{\mathcal{D}},\left(d_{r}^{\mathcal{A}}\right)_{r \in \mathcal{A}},\left(d_{r}^{\mathcal{D}}\right)_{r \in \mathcal{D}}\right\rangle$.

Risk profiles can model highly non-uniform network behaviour with extreme and diverse stress levels. For instance, consider two resources $r$ and $r^{\prime}$, with delays $d_{r}$ and $d_{r^{\prime}}$, controlled by the angel. The way that the angel influences $r$ and $r^{\prime}$ may be very different: it may be the case that $d_{r}^{\mathcal{A}}=\left\lfloor\sqrt{d_{r}}\right\rfloor$ while $d_{r^{\prime}}^{\mathcal{A}}=\left\lfloor\ln d_{r^{\prime}}\right\rfloor$.

Some concrete instances of abnormal (angel and daemon) uniform delay functions are considered and the resulting situations (world views) are analysed using the notion of Weltanschauung. A Weltanschauung defines one uniform type of stress for the angel and another uniform stress for the daemon. The set of Weltanschauungs $\mathfrak{W}$ that are used in the paper are formalised below:

In the first classification the angel and daemon have two possible sensitivities $^{2}$ with respect to the environment: an extreme sensitivity (denoted by $\mathbb{E}$ )

\footnotetext{
${ }^{2}$ In this paper the words sensitivity and moral are used in a mathematical context which, nevertheless, mimics the usual meaning of these words. In [7] sensitive is defined as quick to detect, respond to, or be affected by slight changes, signals, or influences. An alternative meaning (especially well-adapted to the daemon) is easily offended or upset. It is assumed that angel and daemon act instantaneously on the environment.
} 
or a moderate one (denoted by $\mathbb{M}$ ). The set $\mathfrak{W}$ combines these sensitivity types. The set of joint sensitivities is $\mathbb{S}=\{\mathbb{E}-\mathbb{E}, \mathbb{E}-\mathbb{M}, \mathbb{M}-\mathbb{E}, \mathbb{M}-\mathbb{M}\}$. At a second level, both the angel and the daemon have a joint psychological view of the environment called here the moral ${ }^{3}$. A moral is associated with both, the angel and the daemon conjointly; the set of morals formed by the basic types is:

$$
\mathrm{M}=\{\text { Crash, Benevolent, Polarized, Schizophrenic }\}
$$

Formally the set of Weltanschauungs is $\mathfrak{W}=\mathbb{S} \times \mathrm{M}$ and a Weltanschauung is written as $\mathfrak{w} \in \mathfrak{W}$. Depending on the sensitivity and moral, the angel and the daemon stress the delay functions of a resource in the following way:

\begin{tabular}{|l|l|l|l|l|}
\hline & Crash & Benevolent & Polarized & Schizophrenic \\
\hline $\mathbb{E}-\mathbb{E}$ & $d_{r}^{\mathcal{A}}=\infty$ & $d_{r}^{\mathcal{A}}=0$ & $d_{r}^{\mathcal{A}}=0$ & $d_{r}^{\mathcal{A}}=\infty$ \\
& $d_{r}^{\mathcal{D}}=\infty$ & $d_{r}^{\mathcal{D}}=0$ & $d_{r}^{\mathcal{D}}=\infty$ & $d_{r}^{\mathcal{D}}=0$ \\
\hline $\mathbb{E}-\mathbb{M}$ & $d_{r}^{\mathcal{A}}=\infty$ & $d_{r}^{\mathcal{A}}=0$ & $d_{r}^{\mathcal{A}}=0$ & $d_{r}^{\mathcal{A}}=\infty$ \\
& $d_{r}^{\mathcal{D}}=\beta d_{r}$ & $d_{r}^{\mathcal{D}}=\beta d_{r}$ & $d_{r}^{\mathcal{D}}=\beta d_{r}$ & $d_{r}^{\mathcal{D}}=\beta d_{r}$ \\
\hline $\mathbb{M}-\mathbb{E}$ & $d_{r}^{\mathcal{A}}=\alpha d_{r}$ & $d_{r}^{\mathcal{A}}=\alpha d_{r}$ & $d_{r}^{\mathcal{A}}=\alpha d_{r}$ & $d_{r}^{\mathcal{A}}=\alpha d_{r}$ \\
& $d_{r}^{\mathcal{D}}=\infty$ & $d_{r}^{\mathcal{D}}=0$ & $d_{r}^{\mathcal{D}}=\infty$ & $d_{r}^{\mathcal{D}}=0$ \\
\hline $\mathbb{M}-\mathbb{M}$ & $d_{r}^{\mathcal{A}}=\alpha d_{r}$ & $d_{r}^{\mathcal{A}}=\alpha d_{r}$ & $d_{r}^{\mathcal{A}}=\alpha d_{r}$ & $d_{r}^{\mathcal{A}}=\alpha d_{r}$ \\
& $d_{r}^{\mathcal{D}}=\beta d_{r}$ & $d_{r}^{\mathcal{D}}=\beta d_{r}$ & $d_{r}^{\mathcal{D}}=\beta d_{r}$ & $d_{r}^{\mathcal{D}}=\beta d_{r}$ \\
\hline & $1<\alpha<\infty$ & $0<\alpha<1$ & $0<\alpha<1$ & $1<\alpha<\infty$ \\
& $1<\beta<\infty$ & $0<\beta<1$ & $1<\beta<\infty$ & $0<\beta<1$ \\
\hline
\end{tabular}

A Weltanschauung $\mathfrak{w}$ for the case $\mathbb{E}-\mathbb{E}$ is the list of pairs: $(\infty, \infty),(0,0),(0, \infty)$ and $(\infty, 0)$. When the angel and daemon have a moderate behaviour (case $\mathbb{M}-\mathbb{M})$ we assume that $d_{r}^{\mathcal{A}}=\alpha d_{r} d_{r}^{\mathcal{D}}=\beta d_{r}$. The values that $\alpha$ and $\beta$ take depend on the morals. As we can associate a risk profile to a given $\mathfrak{w} \in \mathfrak{W}$ and as we know how to stress $d_{r}$ into $d_{r}^{\mathcal{A}}$ or $d_{r}^{\mathcal{D}}$, then we can extend the definition of risk profile to incorporate Weltanschauung:

Definition 2. Let $C=\left\langle N, R,\left(w_{i}\right)_{i \in N},\left(d_{r}\right)_{r \in R},\left(A_{i}\right)_{i \in N}\right\rangle$ be a resource allocation game and let $\mathfrak{w} \in \mathfrak{W}$ be a Weltanschauung. A risk profile is a tuple $\mathcal{R}=\left\langle C, \mathcal{A}, \mathcal{D}, f_{\mathcal{A}}, f_{\mathcal{D}}, \mathfrak{w}\right\rangle$ where $\mathcal{A} \cap \mathcal{D}=\emptyset$ and $\mathcal{A} \cup \mathcal{D}=R$.

Example 4. Consider a risk profile for the game Fortran\&MPI_Servers (see Example 3). Suppose that the angel $\mathfrak{a}$ controls servers 1 and 2 (i.e. $\mathcal{A}=\{1,2\}$ ) and the daemon $\mathfrak{d}$ controls servers 3 and 4 (i.e. $\mathcal{D}=\{3,4\}$ ). Assume that both the angel and the daemon have limited capacity to act over the game: for instance $f_{\mathcal{A}}=f_{\mathcal{D}}=1$. Consider the following scenario (where cost functions are again rounded by the ceiling function):

- The angel $\mathfrak{a}$ controls a number of servers: assume that one of these servers fails when put under stress i.e. $d_{r}^{\mathcal{A}}=\infty$.

\footnotetext{
${ }^{3}$ In [7] moral is defined as conforming to accepted standards of behaviour. In this paper the moral determines the joint social behaviour of the angel and the daemon.
} 
- The daemon $\mathfrak{d}$ controls a number of robust servers. However, one server's performance is degraded under stress and so $d_{r}^{\mathcal{D}}=\beta d_{r}$ where $\beta=3 / 2$ (note that $\beta>1$ ).

This situation is a Crash Weltanschauung $\mathfrak{w}=(\infty, 3 / 2)$ of type $\mathbb{E}$ - $\mathbb{M}$ and can be used to describe the risk profile

Stop\&Slow $=\langle$ Fortran\&MPI_Servers, $\{1,2\},\{3,4\}, 1,1,(\infty, 3 / 2)\rangle$.

Example 5. A dual scenario arises when the roles of $\mathfrak{a}$ and $\mathfrak{d}$ are interchanged: $\mathcal{A}=\{3,4\}, \mathcal{D}=\{1,2\}$ and $f_{\mathcal{A}}=f_{\mathcal{D}}=1$. Now the angel $\mathfrak{a}$ increases its delay by a factor $3 / 2$. The daemon $\mathfrak{d}$ can close a service $(\mathfrak{d}$ is delighted with such a possibility). This situation is a Crash Weltanschauung $(3 / 2, \infty)$ with risk profile Slow\&Stop $=\langle$ Fortran\&MPI_Servers, $\{3,4\},\{1,2\}, 1,1,(3 / 2, \infty)\rangle$.

Both scenarios are analysed using game theory below.

\section{Angel-daemon games}

A risk profile $\mathcal{R}$ reflects a strategic situation that can be analysed by an angeldaemon game ${ }^{4}$. In such a game, the stress actions are taken by two active players the angel $\mathfrak{a}$ and the daemon $\mathfrak{d}$. The subset of resources $\mathcal{A}$ in $\mathcal{R}$ is controlled by an agent $\mathfrak{a}$ called the angel. The angel tries to improve the behaviour as much as possible. To do that, a selects a subset $a$ of $f_{\mathcal{A}}$ resources in $\mathcal{A}$. When a resource $r$ is selected (formally $r \in a$ ) it runs under the angelic delay function $d_{r}^{\mathcal{A}}(x)$. The angelic cost $c_{\mathfrak{a}}$ is defined as the (entire) social network cost calculated using updated (stressed) delays. Thus, $c_{\mathfrak{a}}(\sigma) \geq 0$. Dually, $\mathcal{D}$ in $\mathcal{R}$ is controlled by another agent $\mathfrak{d}$ called the daemon $\mathcal{D}$. The daemon tries to make the situation deteriorate as much as possible by choosing $f_{\mathcal{D}}$ resources in $\mathcal{D}$. When $r$ is selected by $\mathfrak{d}$, it has a delay $d_{r}^{\mathcal{D}}(x)$. The cost to the daemon is defined as $c_{\mathfrak{d}}=-c_{\mathfrak{a}}$. Note that the definition of the angel and daemon cost functions give rise to opposite behaviours of $\mathfrak{a}$ and $\mathfrak{d}$ : the goal of $\mathfrak{a}$ is to minimise the overall social cost whereas $\mathfrak{d}$ has the opposite strategy. Below is the formal definition of an angel-daemon game with an associated risk profile:

Definition 3. Given $C=\left\langle N, R,\left(w_{i}\right)_{i \in N},\left(d_{r}\right)_{r \in R},\left(A_{i}\right)_{i \in N}\right\rangle$ and a risk profile $\mathcal{R}=\left\langle C, \mathcal{A}, \mathcal{D}, f_{\mathcal{A}}, f_{\mathcal{D}}, \mathfrak{w}\right\rangle$, the angel-daemon game associated to $\mathcal{R}$ is $\Gamma(\mathcal{R})=$ $\left\langle N \cup\{\mathfrak{a}, \mathfrak{d}\},\left(A_{i}\right)_{i \in N}, A_{\mathfrak{a}}, A_{\mathfrak{d}},\left(c_{i}\right)_{i \in N}, c_{\mathfrak{a}}, c_{\mathfrak{d}}\right\rangle$ where $A_{\mathfrak{a}}=\left\{a \subseteq \mathcal{A}|| a \mid=f_{\mathcal{A}}\right\}$ and $A_{\mathfrak{d}}=\left\{b \subseteq \mathcal{D}|| b \mid=f_{\mathcal{D}}\right\}$. Given $(a, d) \in A_{\mathfrak{a}} \times A_{\mathfrak{d}}$ the cost function of a resource $r$ is defined as follows.

$$
d_{r}[a, d]= \begin{cases}d_{r}^{\mathcal{A}} & \text { if } r \in a . \\ d_{r}^{\mathcal{D}} & \text { if } r \in d . \\ d_{r} & \text { if } r \notin(a \cup d) .\end{cases}
$$

\footnotetext{
${ }^{4}$ We define a game using the notation given in [6]. A game is a tuple $\Gamma=$ $\left\langle N,\left(A_{i}\right)_{i \in N},\left(c_{i}\right)_{i \in N}\right\rangle$ such that $N$ is the set of players, $A_{i}$ is the set of actions for player $i$ and $c_{i}$ is the cost of player $i$.
} 
Given a strategy profile $\sigma=(s, a, d)$, player $i \in N$ incurs a cost $c_{i}(\sigma)=$ $d_{s_{i}}[a, d]\left(\ell_{s_{i}}(s)\right)$, the angel cost is $c_{\mathfrak{a}}(\sigma)=\sum_{i \in N} c_{i}(\sigma)$ and the daemon cost is $c_{\mathfrak{d}}(\sigma)=-c_{\mathfrak{a}}(\sigma)$.

Example 6. Consider a one player game SimpleCloud in which a cloud user wishes to execute a job $w$ with weight 16 . Two cloud services can execute $w$ (i.e. $R=\{1,2\}$ and $A_{1}=\{1,2\}$ ). The services have delays $1 / 2$ and $1 / 8$, respectively (i.e. $d_{1}=1 / 2$ and $d_{2}=1 / 8$ ). The two possible strategies for job placement are $s_{1}=1$ or $s_{1}=2$. Suppose that SimpleCloud is stressed using a moderate sensitivity (type $\mathbb{M}-\mathbb{M}$ ) Crash Weltanschauung $\mathfrak{w}=(2,2)$ with two associated risk profiles, Angel and Daemon.

In the Angel profile the angel controls both resources, $\mathcal{A}=R$, but can act over only one, $f_{\mathcal{A}}=1$. Since $\mathcal{D}=\emptyset$ (and $f_{\mathcal{D}}=0$ ) the only strategy for $\mathfrak{d}$ is $d=\emptyset$. As $\mathfrak{d}$ cannot "move" the game $\Gamma($ Angel $)$ has only two "active players", the user and the angel $\mathfrak{a}$. If the angel chooses the first service $(a=\{1\})$ then the delays are $d_{1}[\{1\}, \emptyset]=2 * 1 / 2$ and $d_{2}[\{1\}, \emptyset]=1 / 8$; otherwise $a=\{2\}$ and $d_{1}[\{2\}, \emptyset]=1 / 2$ and $d_{2}[\{2\}, \emptyset]=1 / 4$. Game $\Gamma$ (Angel) is

$$
\begin{aligned}
& \mathfrak{a} \\
& \{1\} \quad\{2\} \\
& \text { user } \begin{array}{c|c|c|}
1 & 16,16 & 8,8 \\
\cline { 2 - 3 } & \multicolumn{2}{c|}{\Gamma(\text { Angel })}
\end{array}
\end{aligned}
$$

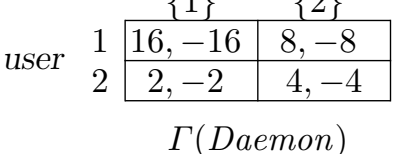

The strategy $(2,\{1\}, \emptyset)$ is the only PNE in $\Gamma$ (Angel). In this equilibrium, a increases the delay of the slower service while the job is placed on the faster one.

Given Daemon $=\langle$ Small $, \emptyset,\{1,2\}, 0,1, \mathfrak{w}\rangle$, the associated game $\Gamma($ Daemon $)$, has again only two effective players (the user and $\mathfrak{d}) ; \Gamma$ (Daemon) has only one PNE: $(2, \emptyset,\{2\})$. In this case $\mathfrak{d}$ increases the delay of the faster service and job $w$ is also allocated to service 2 .

Example 7. Consider SimpleCloud again (see Example 6). Suppose that a moderate beneficial stress is applied to the game, $\mathfrak{w}=(1 / 2,1 / 2)$. With risk profile Angel ${ }^{\prime}=\langle$ SimpleCloud, $\{1,2\}, \emptyset, 1,0, \mathfrak{w}\rangle$, game $\Gamma\left(\right.$ Angel $\left.^{\prime}\right)$ has a PNE $(2,\{2\}, \emptyset)$. With Daemon ${ }^{\prime}=\langle$ SimpleCloud, $\emptyset,\{1,2\}, 0,1, \mathfrak{w}\rangle$ game $\Gamma\left(\right.$ Daemon $\left.^{\prime}\right)$ has a unique PNE, $(2, \emptyset,\{1\})$.

Example 8. Consider game $\Gamma$ (Stop\&Slow) (from Example 4). Consider a risk profile for Fortran\&MPI_Servers (Example 3) where the set of players is $N \cup$ $\{\mathfrak{a}, \mathfrak{d}\}, f_{\mathcal{A}}=f_{\mathcal{D}}=1 A_{\mathfrak{a}}=\{\{1\},\{2\}\}$ and $A_{\mathfrak{d}}=\{\{3\},\{4\}\}$. Suppose that jobs are allocated using the schedule $s=(3,2,3,4,1)$. The angel $\mathfrak{a}$ closes site $1(a=\{1\})$ while the daemon $\mathfrak{d}$ chooses to deteriorate the performance of site $3(d=\{3\})$. The stressed delay functions $d_{r}[a, d]$ are:

$$
\begin{aligned}
& d_{1}[\{1\},\{3\}]=\infty, \quad d_{2}[\{1\},\{3\}]=d_{2}=1 / 4, \\
& d_{3}[\{1\},\{3\}]=\beta d_{3}=3 / 4 \times 1 / 4, \quad d_{4}[\{1\},\{3\}]=d_{4}=1 / 8
\end{aligned}
$$


The preceding profile is not a PNE because $w_{5}^{\mathrm{F}}$ can improve its situation by moving from server 1 . However, $\sigma=((3,2,2,4,2),\{1\},\{3\})$ is a PNE. We have $c_{1}(\sigma)=4, c_{2}(\sigma)=c_{3}(\sigma)=c_{5}(\sigma)=4, c_{4}(\sigma)=2$ with an associated social cost $c_{\mathfrak{s}}(\sigma)=18$ : in this case $c_{\mathfrak{a}}=18$ and $c_{\mathfrak{d}}=-18$. In order to prove that $\sigma$ is a PNE we need to show that no player in $N \cup\{\mathfrak{a}, \mathfrak{d}\}$ is interested in changing its strategy.

- It is easy to see that no job $i \in N$ can improve their (private) cost $c_{i}$ by moving to another server.

- If $\mathfrak{a}$ changes from $\{1\}$ to $\{2\}$, the new profile is $\tau=((3,2,2,4,2),\{2\},\{3\})$ and the new cost is $c_{2}(\tau)=c_{3}(\tau)=c_{5}(\tau)=\infty$. Clearly, the angel $\mathfrak{a}$ will not make such a move.

- If $\mathfrak{d}$ changes from $\{3\}$ to $\{4\}$, the profile is $\tau^{\prime}=((3,2,2,4,2),\{1\},\{4\})$ and the new daemon delay functions are $d_{3}[\{1\},\{4\}]=1 / 4$ and $d_{4}[\{1\},\{4\}]=$ $3 / 2 \times 1 / 8$. The costs are $c_{1}\left(\tau^{\prime}\right)=3, c_{2}\left(\tau^{\prime}\right)=c_{3}\left(\tau^{\prime}\right)=c_{5}\left(\tau^{\prime}\right)=4, c_{4}\left(\tau^{\prime}\right)=3$. and the social cost $c_{\mathfrak{s}}\left(\tau^{\prime}\right)=18$. As social cost of $\tau^{\prime}$ is the same as the social cost of $\sigma$, the agent $\mathfrak{d}$ does not conduct this move.

Example 9. Consider the game $\Gamma$ (Slow\&Stop) (Example 5) again. As before $\mathcal{A}=\{3,4\}, \mathcal{D}=\{1,2\}$ and $f_{\mathcal{A}}=f_{\mathcal{D}}=1 . \Gamma$ (Slow\&Stop) has no PNE. Proof by case analysis.

- Consider profile $\sigma=(s, a,\{1\})$ where $d_{1}[a,\{1\}]=\infty$. Suppose that there is an $i \in N$ such that $s_{i}=1$ (at least one work is placed on server 1). Profile $\sigma$ cannot be a PNE because work $i$ can be placed elsewhere to reduce the social cost.

- Consider profile $\sigma=(s, a,\{1\})$ where no jobs are allocated to server 1 but there is at least one job allocated to server 2 . The daemon $\mathfrak{d}$ would select server $\{2\}$ (rather than 1) to increase the social cost (to infinity). Thus, we can assume that in a PNE no jobs are placed on servers 1 or 2 .

- Consider a profile $\sigma=(s, a,\{1\})$ with no jobs allocated to servers 1 and 2 . Now server 2 works and is free and so the existing Fortran job prefers to move to server 2 and so we get a contradiction.

Case $(s, a,\{2\})$ is similar.

\section{Snapshots and anonymous pure Nash equilibria}

Now we extend the notion of load of a resource $\ell_{r}(s)$ to obtain information about the situation of $r$ in relation to $\mathfrak{a}$ and $\mathfrak{d}$. We also add the delay function being currently used in this resource to obtain an adequate snapshot of the system occupancy. When we need to make explicit the Weltanschauung we replace $d_{r}[a, d]$ with $d_{r}[a, d, \mathfrak{w}]$.

Definition 4. Let $\Gamma(\mathcal{R})$ be an angel-daemon resource allocation game, $\mathcal{R}=$ $\left\langle C, \mathcal{A}, \mathcal{D}, f_{\mathcal{A}}, f_{\mathcal{D}}, \mathfrak{w}\right\rangle$, with profile $\sigma=(s, a, d)$. For a resource $r \in R$ define two 
properties:

$$
\text { affiliation }=\left\{\begin{array}{l}
\mathfrak{a} \text { if } r \in \mathcal{A} \\
\mathfrak{d} \text { otherwise }
\end{array} \quad \text { selected }=\left\{\begin{array}{l}
\mathrm{y} \text { if } r \in a \cup d \\
\mathrm{n} \text { otherwise }
\end{array}\right.\right.
$$

Property affiliation indicates whether $r$ is controlled by the angel or the daemon. Property selected denotes whether $r$ 's behaviour is abnormal or not (i.e. whether $r$ has been chosen by either angel or daemon). A snapshot is a tuple $\delta(\sigma, \mathfrak{w})=$ $\left(\delta_{1}(\sigma, \mathfrak{w})|\cdots| \delta_{k}(\sigma, \mathfrak{w})\right)$ which provides information about the current state of each of the resources where $\delta_{r}(\sigma, \mathfrak{w})=\left(\right.$ affiliation $\left._{\text {selected }}, \ell_{r}(s), d_{r}[a, d, \mathfrak{w}]\right)$

Snapshots provide a clear picture of (i) the occupancy of resources, (ii) the strategies of $\mathfrak{a}$ and $\mathfrak{d}$, and (iii) the delay functions applicable to each resource. When the context is known we abbreviate the notation of a snapshot to $\delta(\mathfrak{w})$.

Example 10. Consider Unit $_{2,4, d}$ under a risk profile $\mathcal{A}=\{1,2\}, \mathcal{D}=\{3,4\}$ and $f_{\mathcal{A}}=f_{\mathcal{D}}=1$. Given $\sigma=(s, a, d)=((2,4),\{1\},\{4\})$ and $\mathfrak{w}=(\infty, \infty)$, the snapshot is $\delta(\sigma,(\infty, \infty))=\left(\mathfrak{a}_{\mathrm{y}}, 0, \infty\left|\mathfrak{a}_{\mathrm{n}}, 1, d x\right| \mathfrak{d}_{\mathrm{n}}, 0, d x \mid \mathfrak{d}_{\mathrm{y}}, 1, \infty\right)$

Lemma 2. Let $\mathcal{R}=\left\langle\right.$ Unit $\left._{n, k, d}, \mathcal{A}, \mathcal{D}, f_{\mathcal{A}}, f_{\mathcal{D}}, \mathfrak{w}\right\rangle$, be a risk profile associated with a unit resource allocation game and Weltanschauung $\mathfrak{w}$ and let $\sigma=(s, a, d)$, $\sigma^{\prime}=\left(s^{\prime}, a^{\prime}, d^{\prime}\right)$ be two strategy profiles of the angel-daemon game $\Gamma(\mathcal{R})$. Then $\delta(\sigma, \mathfrak{w})=\delta\left(\sigma^{\prime}, \mathfrak{w}\right)$ iff $a=a^{\prime}$ and $d=d^{\prime}$ and there is a permutation $\pi$ of $\{1, \ldots, n\}$ such that $\pi\left(L_{r}(s)\right)=L_{r}\left(s^{\prime}\right)$ for any $1 \leq r \leq k$.

Proof. Define $R=\{1, \ldots, k\}$ and $N=\{1, \ldots, n\}$. Observe that $\delta(\sigma, \mathfrak{w})=$ $\delta\left(\sigma^{\prime}, \mathfrak{w}\right)$ implies that $a=a^{\prime}$ and $d=d^{\prime}$ and that for any $r \in R \cdot \ell_{r}(\sigma)=\ell_{r}\left(\sigma^{\prime}\right)$. Therefore, for any $r \in R$ the number of players in $N$ that select $r$ is the same for $s$ and $s^{\prime}$. Thus there is a bijection between $L_{r}(s)$ and $L_{r}\left(s^{\prime}\right)$. The permutation $\pi$ is obtained by composing the bijection defined for each resource. Given $\mathcal{R}=\left\langle C, \mathcal{A}, \mathcal{D}, f_{\mathcal{A}}, f_{\mathcal{D}}, \mathfrak{w}\right\rangle$, and strategies $\sigma=(s, a, d), \sigma^{\prime}=\left(s^{\prime}, a^{\prime}, d^{\prime}\right)$ with $a=a^{\prime}$ and $d=d^{\prime}$ such that there exists a permutation $\pi: N \rightarrow N$ satisfying $\pi\left(L_{r}(s)\right)=L_{r}\left(s^{\prime}\right)$ for $r \in R$, then $\delta(\sigma, \mathfrak{w})=\delta\left(\sigma^{\prime}, \mathfrak{w}\right)$.

Let $\pi$ be a permutation fulfilling the conditions given in the lemma. As we have $\pi\left(L_{r}(s)\right)=\left\{\pi(i) \mid s_{i}=\{r\}\right\}=\left\{j \mid s_{j}^{\prime}=\{r\}\right\}$, for every $i$ there exists $j$ such that $\pi(i)=j$ and $s_{i}=s_{j}^{\prime}=\{r\}$. Take $i$ and $r$ such that $s_{i}=s_{\pi(i)}=\{r\}$, then

$$
c_{i}(\sigma)=d_{r}[a, d, \mathfrak{w}]\left(\ell_{r}(s)\right)=d_{r}\left[a^{\prime}, d^{\prime}, \mathfrak{w}\right]\left(\ell_{r}\left(s^{\prime}\right)\right)=c_{\pi(i)}\left(\sigma^{\prime}\right)
$$

As $\pi$ is a permutation $c_{\mathfrak{a}}(\sigma)=\sum_{i \in N} c_{i}(\sigma)=\sum_{i \in N} c_{\pi(i)}\left(\sigma^{\prime}\right)=c_{\mathfrak{a}}\left(\sigma^{\prime}\right)$ and similarly for $\mathfrak{d}$. The conditions on cost follow. Finally, given $\mathcal{R}, s$ and $s^{\prime}$ such that $a=a^{\prime}$ and $d=d^{\prime}$, for any $r$ the parts affiliation selected and $d_{r}[a, d, \mathfrak{w}]$ coincide in $\delta_{r}(\sigma, \mathfrak{w})$ and in $\delta_{r}\left(\sigma^{\prime}, \mathfrak{w}\right)$. Finally, as $\pi\left(L_{r}(s)\right)=L_{r}\left(s^{\prime}\right)$, we have $\ell(s)=\ell\left(s^{\prime}\right)$ and both strategies have the same snapshot.

Lemma 3. Given $\Gamma(\mathcal{R})$ for $\mathcal{R}=\left\langle\right.$ Unit $\left._{n, k, d}, \mathcal{A}, \mathcal{D}, f_{\mathcal{A}}, f_{\mathcal{D}}, \mathfrak{w}\right\rangle$, and $\sigma=(s, a, d)$, $\sigma^{\prime}=\left(s^{\prime}, a, d\right)$ such that $\delta(\sigma, \mathfrak{w})=\delta\left(\sigma^{\prime}, \mathfrak{w}\right)$ then $\sigma$ is a PNE iff $\sigma^{\prime}$ is a PNE. 
Proof. There exists $\pi$ such that the role of player $i$ in $s$ is mapped into the role of player $\pi(i)=j$ in $s^{\prime}$. We can imagine $j$ as an alias of $i$. Imagine that $i$ is interested in changing from $r$ to $\hat{r}$, that is change $s_{i}=\{r\}$ into $\hat{s}_{i}=\{\hat{r}\}$, because $c_{i}\left(\sigma_{-i}, \hat{s}_{i}\right)<c_{i}(\sigma)$. Note that $\ell_{\hat{r}}(\sigma)=\ell_{\hat{r}}\left(\sigma^{\prime}\right)$ and $\ell_{\hat{r}}\left(\sigma_{-i}, \hat{s}_{i}\right)=\ell_{\hat{r}}(\sigma)+1$. As $s_{i}=s_{\pi(i)}^{\prime}=\{r\}$, defining $\hat{s}_{\pi(i)}=\{\hat{r}\}$ we have the loads $\ell_{\hat{r}}\left(\sigma_{-\pi(i)}^{\prime}, \hat{s}_{\pi(i)}\right)=$ $\ell_{\hat{r}}\left(\sigma^{\prime}\right)+1=\ell_{\hat{r}}\left(\sigma_{-i}, \hat{s}_{i}\right)$ and

$$
c_{\pi(i)}\left(\sigma_{-\pi(i)}^{\prime}, \hat{s}_{\pi(i)}\right)=d_{\hat{r}}[a, b]\left(\ell\left(\sigma_{-\pi(i)}^{\prime}, \hat{s}_{\pi(i)}\right)\right)=c_{i}\left(\sigma_{-i}, \hat{s}_{i}\right)
$$

and player $\pi(i)$ has an interest in changing the strategy. Suppose that $\mathfrak{a}$ is interested in changing in $\sigma=(s, a, d)$ from $a$ to $\hat{a}$ because $c_{\mathfrak{a}}\left(s_{-\mathfrak{a}}, \hat{a}\right)<c_{\mathfrak{a}}(s)$. Note that $\ell_{r}(s)=\ell_{r}\left(s_{-\mathfrak{a}}, \hat{a}\right)$ for any $r \in R$, therefore

$$
c_{\mathfrak{a}}\left(s_{-\mathfrak{a}}, \hat{a}\right)=\sum_{r \in R} \ell_{r}(s) d_{r}[\hat{a}, d]\left(\ell_{r}(s)\right)=c_{\mathfrak{a}}\left(s_{-\mathfrak{a}}^{\prime}, \hat{a}\right)
$$

and $\mathfrak{a}$ has an interest in changing also in $s^{\prime}$. The daemon has similar behaviour.

In snapshots resource loads are important but the player of the load is not. Thus, by lemmas 2 and 3, snapshots describe Nash equilibria in an "anonymous way". This idea is at the root of the following definition.

Definition 5. A snapshot $\delta$ is called an anonymous Nash equilibrium iff there exists a PNE $\sigma$ such that $\delta(\sigma)=\delta$.

The following lemma demonstrates that, even in simple cases, the existence of a set of resources under the control of a daemon prohibits the existence of a pure Nash equilibrium.

Lemma 4. For any $\mathfrak{w} \in \mathfrak{W}$, the game $\Gamma\left(\mathcal{R}_{\mathfrak{w}}\right)$ corresponding to the profile $\mathcal{R}_{\mathfrak{w}}=$ $\left\langle\right.$ Unit $\left._{1,2, d}, \emptyset,\{1,2\}, 0,1, \mathfrak{w}\right\rangle$ has no pure Nash equilibria.

Proof. The game Unit $t_{1,2, d}$ without an angel or daemon has two Nash equilibria, namely, $s_{1}=\{1\}$ or $s_{1}=\{2\}$. The introduction of a daemon changes the situation completely. Now there are no anonymous Nash equilibria.

Define $R=\{1,2\}$. As in $\mathcal{R}_{\mathfrak{w}}$ we have $\mathcal{A}=\emptyset, \mathcal{D}=R, f_{\mathcal{A}}=0$ and $f_{\mathcal{D}}=1$, by symmetry we have to consider only the snapshots $\left(\mathfrak{d}_{\mathrm{y}}, 1, d^{\mathcal{D}}(x) \mid \mathfrak{d}_{\mathrm{n}}, 0, d(x)\right)$, $\left(\mathfrak{d}_{\mathrm{y}}, 0, d^{\mathcal{D}}(x) \mid \mathfrak{d}_{\mathrm{n}}, 1, d(x)\right)$. As usual $d(x)=d x$. The possible values for a demonic delay function are $d^{\mathcal{D}}(x)=\infty, d^{\mathcal{D}}(x)=0, d^{\mathcal{D}}(x)=\alpha d x$ with $1<\alpha<\infty$ and $d^{\mathcal{D}}(x)=\alpha d x$ with $0<\alpha<1$. Let us consider each case separately.

(1) When $d^{\mathcal{D}}(x)=\infty$ we have to consider two snapshots, $\left(\mathfrak{d}_{\mathrm{y}}, 1, \infty \mid \mathfrak{d}_{\mathrm{n}}, 0, d x\right)$ and $\left.\left(\mathfrak{d}_{\mathrm{y}}, 0, \infty \mid \mathfrak{D}_{\mathrm{n}}, 1, d x\right)\right\}$. The first one is not an anonymous Nash because the player located at $r=1$ has an interest in moving to $r=2$ improving the cost from $\infty$ to $2 d$. The second one is not an anonymous Nash because $\mathfrak{d}$ has interest to abandon $r=1$ and select (destroy) $r=2$ increasing the delay from $d$ to $\infty$.

(2) When $d^{\mathcal{D}}(x)=0$ neither $\left(\mathfrak{d}_{\mathrm{y}}, 1,0 \mid \mathfrak{d}_{\mathfrak{n}}, 0, d x\right)$ nor $\left(\mathfrak{d}_{\mathrm{y}}, 0,0 \mid \mathfrak{d}_{\mathfrak{n}}, 1, d x\right)$ are anonymous Nash. In the first one $\mathfrak{d}$ has interest to select the second resource 
because nobody is using it. In the second one, the user of the resource 2 has interest to use resource 1.

(3) When $d^{\mathcal{D}}(x)=\alpha d x$ with $1<\alpha<\infty$, the analysis of the snapshots $\left(\mathfrak{d}_{\mathrm{y}}, 1, \alpha d x \mid \mathfrak{d}_{\mathrm{n}}, 0, d x\right)$ and $\left(\mathfrak{d}_{\mathrm{y}}, 0, \alpha d x \mid \mathfrak{o}_{\mathrm{n}}, 1, d x\right)$ is similar to the case $d^{\mathcal{D}}(x)=\infty$. (4) When $d^{\mathcal{D}}(x)=\alpha d x$ with $0<\alpha<1$ the analysis is similar to $d^{\mathcal{D}}(x)=0$.

\section{On pure Nash equilibria}

As a consequence of the preceding lemma we have the following theorem

Theorem 1. There are tuples $\left\langle\right.$ Unit $\left._{n, k, d}, \mathcal{A}, \mathcal{D}, f_{\mathcal{A}}, f_{\mathcal{D}}\right\rangle$ such that for any $\mathfrak{w} \in$ $\mathcal{W}$, the profile $\mathcal{R}_{\mathfrak{w}}=\left\langle\right.$ Unit $\left._{n, k, d}, \mathcal{A}, \mathcal{D}, f_{\mathcal{A}}, f_{\mathcal{D}}, \mathfrak{w}\right\rangle$ describes a game $\Gamma\left(\mathcal{R}_{\mathfrak{w}}\right)$ with no pure Nash equilibria.

However, when the game has an angel but no daemon $\mathfrak{d}$, there are anonymous Nash equilibria (see below):

Example 11. Given $\mathcal{R}_{\mathfrak{w}}=\left\langle\right.$ Unit $\left._{1,2, d},\{1,2\}, \emptyset, 1,0, \mathfrak{w}\right\rangle$ for any $\mathfrak{w} \in \mathfrak{W}$. It is easy to see that, $\left(\mathfrak{a}_{\mathrm{y}}, 0, d^{\mathcal{A}}(x) \mid \mathfrak{a}_{\mathrm{n}}, 1, d x\right)$ is an anonymous pure Nash equilibrium when $d^{\mathcal{A}}(x)=\infty$ or $d^{\mathcal{A}}(x)=\alpha d x$ when $1<\alpha<\infty$ (this corresponds to a crash moral). The following snapshot $\left(\mathfrak{a}_{\mathrm{y}}, 1, d^{\mathcal{A}}(x) \mid \mathfrak{a}_{\mathrm{n}}, 0, d x\right)$ is an anonymous Nash when $d^{\mathcal{A}}(x)=0$ or $d^{\mathcal{A}}(x)=\alpha d x$ when $0<\alpha<1$ (under a benevolent moral)

When Unit $_{n, k, d}$ is under control of both $\mathfrak{a}$ and $\mathfrak{d}$ a variety of situations can arise (as in the following example):

Example 12. Consider $\mathcal{R}_{\mathfrak{w}}=\left\langle\right.$ Unit $\left._{2,4, d},\{1,2\},\{3,4\}, 1,1, \mathfrak{w}\right\rangle$ for any $\mathfrak{w}$ having extreme sensitivity. Under Crash there is no pure Nash. In this moral, no player wishes to allocate work to resources selected by $\mathfrak{a}$ or $\mathfrak{d}$ (infinite delay). The snapshot $\left(\mathfrak{a}_{\mathrm{y}}, 0, \infty\left|\mathfrak{a}_{\mathrm{n}}, 1, d x\right| \mathfrak{d}_{\mathrm{n}}, 1, d x \mid \mathfrak{d}_{\mathrm{y}}, 0, \infty\right)$ is not an anonymous Nash because $\mathfrak{d}$ would select resource 3 . Similarly $\left(\mathfrak{a}_{\mathrm{y}}, 0, \infty\left|\mathfrak{a}_{\mathrm{n}}, 0, d x\right| \mathfrak{d}_{\mathrm{n}}, 0, d x \mid\right.$ $\left.\mathfrak{d}_{\mathrm{y}}, 2, \infty\right)$ is not an anonymous pure Nash. The snapshot $\left(\mathfrak{a}_{\mathrm{y}}, 0, \infty\left|\mathfrak{a}_{\mathrm{n}}, 2, d x\right|\right.$ $\left.\mathfrak{d}_{\mathrm{n}}, 0, d x \mid \mathfrak{d}_{\mathrm{y}}, 0, \infty\right)$ is not stable because one player has an interest in moving to resource 3 . When the moral is benevolent $\left(\mathfrak{a}_{\mathrm{y}}, 2,0\left|\mathfrak{a}_{\mathfrak{n}}, 0, d x\right| \mathfrak{d}_{\mathfrak{n}}, 0, d x \mid \mathfrak{d}_{\mathrm{y}}, 0,0\right)$ is the only anonymous pure Nash equilibrium. When the moral is polarised the anonymous Nash is $\left(\mathfrak{a}_{\mathrm{y}}, 2,0\left|\mathfrak{a}_{\mathfrak{n}}, 0, d x\right| \mathfrak{d}_{\mathfrak{n}}, 0, d x \mid \mathfrak{d}_{\mathrm{y}}, 0, \infty\right)$. Finally, when the moral is schizophrenic there is no Nash. In this case no player chooses a resource selected by $\mathfrak{a}$ (infinite delay). As the resource selected by $\mathfrak{d}$ has delay 0 players will move to this resource but $\mathfrak{d}$ will subsequently select the other resource.

When there are enough resources to locate all the players on the angelic side, sometimes there are Nash equilibria.

Theorem 2. If $\mathcal{R}_{\mathfrak{w}}=\left\langle\right.$ Unit $\left._{n, k, d}, \mathcal{A}, \mathcal{D}, f_{\mathcal{A}}, f_{\mathcal{D}}, \mathfrak{w}\right\rangle$ such that $\mathfrak{w}$ has moral Crash or Polarized, $f_{\mathcal{A}}+n \leq \# \mathcal{A}$, then $\Gamma\left(\mathcal{R}_{\mathfrak{w}}\right)$ has always a pure Nash equilibrium.

Proof. Given $N=\{1, \ldots, n\}$, When there is enough place on the angelic side and no player $i \in N$ is tempted to use a resource in the demonic side the angel and the players $i \in N$ are in agreement. 
We consider separately the morals Crash and Polarized. In the case of a Crash moral, $d_{r}^{\mathcal{A}}=\alpha d$ with $1<\alpha \leq \infty$ and $d_{r}^{\mathcal{D}}=\beta d$ with $1<\beta \leq \infty$. Both, $\mathfrak{a}$ and $\mathfrak{d}$ strictly increases de delay (degradates the performance) of $f_{\mathcal{A}}$ and $f_{\mathcal{D}}$ servers respectivelly. Consider the possibilities offered to the broker in such a risk profile. Whenever possible, the broker allocates jobs in undegradated servers. As $n \leq \# \mathcal{A}-f_{\mathcal{A}}$ the angel has enough undegrated services to allocate the $n$ jobs. The broker spreads the $n$ jobs in different servers and the social cost is $d n$. Let us see that such an allocation is a PNE.

- As a job $i$ is located in non-degradeted server, job $i$ is not interested to move into one of the $f_{\mathcal{A}}+f_{\mathcal{D}}$ servers because delay increases.

- The angel $\mathfrak{a}$ having selected $f_{\mathcal{A}}$ servers has no interest no change the initial choice. For instance if it degradates a site containing a job, the social cost becomes $(n-1) d+\alpha d$ and therefore it increases in $(\alpha-1) d>0$.

- Any change in $\mathfrak{d}$ makes no change in the social cost.

Consider the case of a Polarized moral. In this case $d_{r}^{\mathcal{A}}=\alpha d$ with $0 \leq \alpha<1$ and $d_{r}^{\mathcal{D}}=\beta d$ with $1<\beta \leq \infty$. The angel $\mathfrak{a}$ improves $f_{\mathcal{A}}$ servers and $\mathfrak{d}$ degradates $f_{\mathcal{D}}$ servers. Initially the broker could locate the jobs into the untouched serves from the angelic side, this give a social cost of $d n$. This allocation is far from to be a PNE. For instance, $\mathfrak{a}$ will select and improve $\min \left\{n, f_{\mathcal{A}}\right\}$ servers containing a job and the social cost improves to $\alpha d \min \left\{n, f_{\mathcal{A}}\right\}+\left(n-\min \left\{n, f_{\mathcal{A}}\right\}\right) d$. In many cases this situation is not yet a PNE. For instance, in the case of $\alpha$ being "really small", the jobs remaining in normal servers have interes to move to a server improved by the angel. Suppopse that such one server contains $x$ jobs with cost $\alpha d x$. A job in an a normal server has cost $d$. This job has interest to move into an improved server if $\alpha d(x+1)<d$. This process will continue until a PNE is reached.

\section{Brokering}

The use of risk profiles and Weltanschauungs may be extended from modelling stressful grid environments to deriving resource allocation strategies for brokers. Given an resource allocation problem $C$ and an adequate risk profile $\mathcal{R}$ of a web environment it is possible to determine if there is a pure Nash equilibrium in $\Gamma(\mathcal{R})$; if so the PNE $(s, a, d)$ with optimal cost can be sent from the broker to each player. Even in cases where there are no PNE the risk profiles for the environment still provide an abstract description of web interactions which may provide insights into which resources the broker should utilise.

Different brokers can have different criteria about the adequacy of risk profiles and which parameters are critical. Depending on the brokers, different criteria for optimality can be adopted. The following example illustrates how risk profiles might be utilised by brokers.

Example 13. Consider the allocation problem given by a Unit ${ }_{3,4, d}$. With no information about network stress players should (eventually) choose any Nash 
equilibrium. A Nash equilibrium is obtained allocating different players in different resources. For instance $s_{1}=1, s_{2}=2, s_{3}=3$ is a Nash.

Suppose that the allocation problem for Unit $_{3,4, d}$ is submitted to a broker. The current stress situation about the resources is known by the broker and is summarised in the risk profile $\mathcal{R}=\left\langle\right.$ Unit $\left._{3,4, d},\{1,2\},\{3,4\}, 1,1, \mathfrak{w}\right\rangle$ with a benevolent Weltanschauung $\mathfrak{w}=(1 / 2,1 / 2)$. In $\Gamma(\mathcal{R})$ the snapshot

$$
\left(\mathfrak{a}_{\mathrm{y}}, 1, d x / 2\left|\mathfrak{a}_{\mathrm{n}}, 0, d x\right| \mathfrak{o}_{\mathrm{y}}, 1, d x / 2 \mid \mathfrak{o}_{\mathrm{n}}, 1, d x\right)
$$

is an anonymous Nash. The broker forwards to the four players any allocation consistent with the anonymous Nash. For instance $s_{1}=1, s_{2}=3$ and $s_{3}=4$ is a possible allocation and $s_{1}=3, s_{2}=4, s_{3}=1$ is other possible suggestion. Note that:

1. In a given allocation some players will have better outcomes than others; for example, in the first allocation players 1 and 2 have delay $d / 2$ but player 3 has delay $d$.

2. In different allocations players can have different delays. For example, player 3 has delays $d$ and $d / 2$ in the first and second allocations, respectively.

In this case the behaviour of the broker is clear: it should never propose the allocation $s_{1}=1, s_{2}=2, s_{3}=3$ since here is no snapshot consistent with this allocation which corresponds to an anonymous Nash. If resources 1 and 2 are selected, the angel $\mathfrak{a}$ can improve one of them, say the first, giving the initial snapshot $\left(\mathfrak{a}_{\mathrm{y}}, 1, d x / 2\left|\mathfrak{a}_{\mathrm{n}}, 1, d x\right| \cdots, 1, \cdots \mid \ldots, 0, \ldots\right)$. $\mathfrak{d}$ will elect to damage the allocation by choosing resource 4 giving snapshot $\left(\mathfrak{a}_{\mathrm{y}}, 1, d x / 2\left|\mathfrak{a}_{\mathrm{n}}, 1, d x\right|\right.$ $\mathfrak{d}_{\mathrm{n}}, 1, d x \mid \mathfrak{d}_{\mathrm{y}}, 0, d x / 2$ ) which does not correspond to an anonymous Nash (player 3 now has an incentive to allocate into resource 4 ).

\section{Discussion}

Conventionally the behaviour of a web service is captured by treating the service in isolation. For example, a service level agreement (SLA) might provide information about the expected behaviour of a service. In this paper an alternative view of web services is presented; here the behaviour of a set of services within a stressed web environment is modelled by a strategic angel daemon game. Two different abstractions for modelling stressed web environments are presented, namely, risk profiles and Weltanschauungs. Risk profiles partition web services into angel and daemon sets. Weltanschauungs consider the various scenarios that can arise when angels and daemons have uniform abnormal delay functions at a number of sensitivities. The use of risk profiles and Weltanschauungs raises a number of questions about how the model should be interpreted in a concrete situation:

1. How can a set of services be partitioned into angel and daemon controlled sets?

2. How can a service's performance improve under stress? 
3. How can abnormal bounds for the angel and the daemon be set?

Perhaps one way to interpret the angel/daemon partition is by means of a cost model: sets of low cost services are liable to be severely affected by stressed environments - such sets of services may be considered to be under the control of a daemon. On the other hand expensive services may be responsive even when the surrounding environment becomes stressed. Note that some services may be implemented on elastic clouds - as demand increases a service may call upon more servers to facilitate ongoing requests. In this way it may be the case that behaviour in abnormal stressed conditions may even be better than under normal conditions. However, it seems likely that the choice of the abnormal bound parameters would have to be made on the basis of experimental evidence.

The work reported in this paper provides insights into the dynamic behaviour of sets of services embedded within a (stressed) web environment. Treating network stress as a non-cooperative game clearly reflects the experience of web users and brokers.

\section{References}

1. Babaioff, M; Kleinberg, K; Papadimitriou; C: Congestion games with malicious players, ACM Conference on Electronic Commerce, 2007, 103-112.

2. Baraglia, R; Laforenza, D.; Ferrini, R; Adami, D; Giordano, S; Yahyapour, R: A study on network resources management in grids. CoreGRID-IW, 2006, 213-224.

3. Gabarro, J; García, A.; Clint, M; Stewart, A; Kilpatrick, P: Bounded Site Failures: an Approach to Unreliable Grid Environments. In Making Grid Works edited by M. Danelutto, P. Fragopoulou, V. Getov. Springer Verlag, 2007, 175-187.

4. Gabarro, J; García, A; Serna, M:, On the Complexity of Equilibria Problems in Angel-Daemon Games. In Computing and Combinatorics, 14th Annual International Conference, COCOON 2008, Springer Verlag, LNCS 5092, 2008, 31-40.

5. Gabarro, J; García, Serna, M; Stewart, A; Kilpatrick, P: Analysing Orchestrations with Risk Profiles and Angel daemon Games. In Grid Computing Achievements and Prospects. Edited by S. Gorlatch, P. Fragopoulou, Th. Priol. Springer Verlag, 2008, 121-132.

6. Osborne, M; Rubinstein, A: A Course on Game Theory, MIT Press, 1994.

7. Oxford Dictionaries. http://www . askoxford. com/?view=uk

8. Nisan, N; Roughgarden,T; Tardos, E; Vazirani, V: Algorithmic Game Theory Cambridge, 2007.

9. Penn, M; Maria Polukarov, M; Tennenholtz, M.: Congestion games with failures, ACM Conference on Electronic Commerce, 2005, 259-268.

10. Penn, M; Maria Polukarov, M; Tennenholtz, M.: Congestion games with loaddependent failures: identical resources, ACM Conference on Electronic Commerce, 2007, 210-217.

11. Koutsoupias, E; Papadimitriou, C: Worst-case Equilibria, STACS-1999,LNCS 1563 404-413.

12. Rosenthal, R.: A class of games possessing pure-strategy Nash equilibria. Int. J. Game Theory, 2, 1973, 65-67, 